\title{
(2) OPEN ACCESS \\ Implementation challenges to patient safety in Guatemala: a mixed methods evaluation
}

\author{
Bria J Hall (D) , ${ }^{1}$ Melany Puente, ${ }^{1}$ Angie Aguilar, ${ }^{2}$ Isabelle Sico, ${ }^{3}$ \\ Monica Orozco Barrios, ${ }^{4}$ Sindy Mendez, ${ }^{2}$ Joy Noel Baumgartner, ${ }^{3}$ \\ David Boyd, ${ }^{3}$ Erwin Calgua, ${ }^{4}$ Randall Lou-Meda, ${ }^{5}$ Carla C Ramirez, ${ }^{2}$ \\ Ana Diez, ${ }^{2}$ Astrid Tello, ${ }^{2}$ J Bryan Sexton, ${ }^{6}$ Henry Rice ${ }^{1,3}$
}

- Additional supplemental material is published online only. To view, please visit the journal online (http://dx. doi. org/10.1136/bmjgs-2020012552).

'Department of Surgery, Duke University School of Medicine Durham, North Carolina, USA ${ }^{2}$ Roosevelt Hospital of Guatemala, Guatemala City, Guatemala

${ }^{3}$ Duke Global Health Institute, Durham, North Carolina, USA ${ }^{4}$ University of San Carlos of Guatemala, Guatemala City, Guatemala

${ }^{5}$ Pediatric Nephrology Unit/ Fundanier, Roosevelt Hospital of Guatemala, Guatemala City, Guatemala

${ }^{6}$ Duke Center for Healthcare

Safety and Quality, Duke University Health System, Durham, North Carolina, USA

\section{Correspondence to}

Dr Henry Rice, Department of Surgery, Duke University School of Medicine, Durham, NC 27710, USA;

henry.rice@duke.edu

Received 13 October 2020 Accepted 17 May 2021

\section{Check for updates}

(C) Author(s) (or their employer(s)) 2021. Re-use permitted under CC BY-NC. No commercial re-use. See rights and permissions. Published by BMJ.

To cite: Hall $B J$, Puente $M$ Aguilar A, et al. BMJ Qual Saf Epub ahead of print: [please include Day Month Year]. doi:10.1136/

bmjqs-2020-012552

\begin{abstract}
Background Little is known about factors affecting implementation of patient safety programmes in low and middle-income countries. The goal of our study was to evaluate the implementation of a patient safety programme for paediatric care in Guatemala.

Methods We used a mixed methods design to examine the implementation of a patient safety programme across 11 paediatric units at the Roosevelt Hospital in Guatemala. The safety programme included: (1) tools to measure and foster safety culture, (2) education of patient safety, (3) local leadership engagement, (4) safety event reporting systems, and (5) quality improvement interventions. Key informant staff $(n=82)$ participated in qualitative interviews and quantitative surveys to identify implementation challenges early during programme deployment from May to July 2018, with follow-up focus group discussions in two units 1 year later to identify opportunities for programme modification. Data were analysed using thematic analysis, and integrated using triangulation, complementarity and expansion to identify emerging themes using the Consolidated Framework for Implementation Research. Salience levels were reported according to coding frequency, with valence levels measured to characterise the degree to which each construct impacted implementation.

Results We found several facilitators to safety programme implementation, including high staff receptivity, orientation towards patient-centredness and a desire for protocols. Key barriers included competing clinical demands, lack of knowledge about patient safety, limited governance, human factors and poor organisational incentives. Modifications included use of tools for staff recognition, integration of education into error reporting mechanisms and designation of trained champions to lead unit-based safety interventions.

Conclusion Implementation of safety programmes in low-resource settings requires recognition of facilitators such as staff receptivity and patientcentredness as well as barriers such as lack of training in patient safety and poor organisational incentives. Embedding an implementation analysis during programme deployment allows for programme modification to enhance successful implementation.
\end{abstract}

\section{INTRODUCTION}

The burden of medical errors in low and middle-income countries (LMICs) is high, with two-thirds of health-related adverse events occurring in LMICs. ${ }^{1}$ Protecting patients from avoidable harm is increasingly emphasised within the global health agenda, as evidenced by the World Health Assembly Resolution 55.18, the WHO Patient Safety Programmes and the Lancet Commission on High-Quality Health Care. ${ }^{2-4}$ Remarkable progress has been made over recent years in reduction of hospital-associated infections, use of patient hand-offs and control of adverse drug events, although the majority of this progress has been in high-income settings. ${ }^{5}$ Many safety programmes are often not successfully implemented in LMICs, although the factors which drive successful programme implementation are poorly understood.

Health programme implementation is influenced by many factors, including the local context, external policies and leadership support. ${ }^{6}$ The use of structured frameworks such as the Consolidated Framework for Implementation Research (CFIR) can identify important influencing factors for programme implementation. $^{7-9}$ The CFIR is a meta-theoretical framework that provides a structure of implementation-related constructs that can be applied across a spectrum of settings. ${ }^{8}$ The CFIR comprises 39 constructs organised across five domains, including (1) characteristics of the intervention, (2) outer setting, (3) inner setting, (4) characteristics of individuals involved, and (5) process to achieve implementation. ${ }^{8}$ As many hospitals in low-resource settings around the world continue to incorporate patient safety 


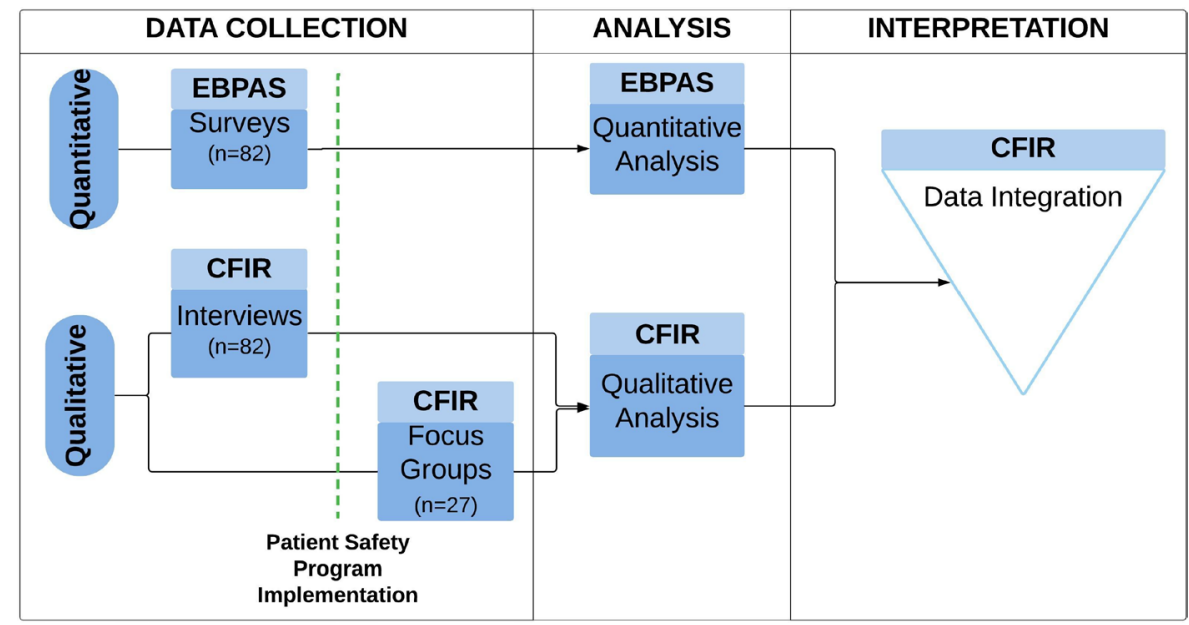

Figure 1 Diagrammatic representation of mixed methods concurrent triangulation design. Semistructured interviews (SSIs) and the Evidence-Based Practice Attitude Scale-36 (EBPAS-36) survey were administered concurrently at the time of the comprehensive patient safety programme implementation. Follow-up focus group discussions (FGDs) in two units were conducted 1 year later to probe for modifications to programme design and implementation. CFIR, Consolidated Framework for Implementation Research.

strategies, implementation research can help identify contextual factors to enhance successful programme implementation. ${ }^{10}$

We have worked for several years to deploy a comprehensive patient safety programme in Guatemala that is based on mutually reinforcing components to foster a strong safety culture, teach patient safety principles and support locally driven quality improvement (QI) initiatives. ${ }^{11-13}$ Using qualitative methods, we previously examined the implementation of this programme in a single perioperative unit. ${ }^{13}$ The objective of our current study was to evaluate implementation using a mixed methods design to identify contextual barriers and facilitators to safety programme implementation across the entire paediatric department at the largest public hospital in Guatemala. We embedded this implementation analysis early during programme deployment to enhance successful implementation.

\section{METHODS}

\section{Study design and research tools}

We used a mixed methods (QUAL+quan) concurrent study design to examine staff views towards the implementation of a patient safety programme across the paediatric department ( $\mathrm{n}=11$ units) at the Roosevelt Hospital in Guatemala City (figure 1). Use of this nomenclature communicates that qualitative findings were considered dominant (QUAL) over quantitative findings (quan), given the value of qualitative analysis in exploring the complex challenges with programme implementation in an LMIC. ${ }^{14}$ Our dominant conceptual framework was drawn from the CFIR and supporting literature. ${ }^{8} 15$

To quantitatively assess staff attitudes towards adoption of a patient safety programme, we used the Evidence-Based Practice Attitude Scale-36 (EBPAS-36) (online supplemental file 1). ${ }^{16}$ The EBPAS-36 measures respondent views across 12 domains. The EBPAS survey has been validated in a number of high-income and middle-income settings, and has been translated and validated in several non-English languages, including Spanish. ${ }^{17-19}$

To qualitatively assess staff views towards implementation, we used semistructured interviews (SSIs). ${ }^{20}$ We conducted SSIs using a 15-question interview tool with questions aligned with CFIR domains as described previously (online supplemental file 2). ${ }^{13}$ Interviews were used to evaluate staff perceptions towards patient safety, as well as barriers and facilitators to programme implementation. Although the SSIs explored all CFIR domains, the interview guide prioritised CFIR constructs based on our prior stakeholder analysis. 1121

One year after programme deployment, we conducted follow-up focus group discussions (FGDs) to explore staff responses to the intervention and identify opportunities to improve the programme or implementation process. Due to time constraints, we limited our FGDs to two larger units (the perioperative and neonatology units). FGDs were coled by a Spanish-speaking local patient safety expert and the senior author with the assistance of an interpreter.

Before programme deployment, all research instruments underwent forward and back translation by a Guatemalan study team member, cultural adaptation and cognitive interviewing using the WHO recommended guidelines. ${ }^{22}{ }^{23}$ We tested all research tools in a single paediatric care unit (paediatric nephrology) to confirm applicability. Deviations between the original and back-translated versions during pilot testing were resolved by consensus between study team members.

All study procedures were exempt by the institutional review board at Duke University. Respondents verbalised consent prior to inclusion in the study and 
were not incentivised for participation. This work was conducted in line with the Consolidated Criteria for Reporting Qualitative Research (online supplemental file 3$){ }^{24}$

\section{Setting}

Roosevelt Hospital in Guatemala City is the largest national public tertiary hospital in Guatemala. We conducted this analysis across the entire paediatric department, which is composed of 12 units and employs more than 400 hospital staff. One unit (paediatric nephrology) was excluded from this evaluation as their unit served as a field site to test our safety programme and research tools, leaving 11 units for this analysis. Most physicians work part-time in the private sector, consistent with common practices across Central America.

\section{Comprehensive patient safety programme}

Our comprehensive patient safety programme has been previously described, ${ }^{13}$ and is defined by five mutually reinforcing components: (1) tools to measure and foster a strong safety culture, (2) education of patient safety principles, (3) governance and leadership engagement, (4) safety event reporting, and (5) unit-tailored QI interventions (online supplemental file 4). ${ }^{25}$ All programme core components are adaptable to support unit-based local processes and needs. ${ }^{26}$ Prior to this intervention, no comprehensive patient safety programme had been used in this hospital. A team of local physicians trained in patient safety $(n=3)$ operated as patient safety leaders to oversee implementation efforts and champions in each unit. We disseminated safety culture assessments to staff in all participating units $(80 \%$ response rate, $\mathrm{n}=308$ participants) as described previously. ${ }^{11}$ Safety culture results were disseminated at the unit and department levels, followed by debriefings to guide QI interventions targeted towards areas of need, such as enhancement of perioperative checklists, standardisation of catheterisation protocols and use of morning huddles.

\section{Participants and data collection}

During early programme implementation, qualitative and quantitative data on implementation challenges were collected from May to July 2018. For SSIs, we used convenience and purposeful sampling to identify key informants $(\mathrm{n}=82)$ in 11 paediatric units. All unit leaders (physician, nursing, other staff) were identified as potential study subjects, with participants limited only by their availability. Two bilingual Guatemalan study team members conducted the interviews in Spanish or English with an average interview length of $17 \mathrm{~min}$ (range 7-65). These same key informants completed the EBPAS-36 survey.

One year after programme implementation, FGDs were held in the perioperative and neonatology units to reassess implementation challenges and identify opportunities for modification of the safety programme itself or the implementation process. To enhance credibility and confirmability, the FGDs incorporated dissemination of SSI results for participant checking. Perioperative FGD length was 59 min and neonatology FGD length was 43 min. Neither patients nor members of the public were involved in this research.

\section{Programme adaptation}

Following data analysis, iterative modifications to the implementation process were made according to implementation challenges. For example, FGDs in the neonatology unit reported low compliance with medical error reporting attributed to knowledge gaps and a 'culture of blame'. These findings led to recommendations for how to modify their error reporting system, which leadership subsequently modified in a 'real-time' fashion.

\section{Data analysis}

\section{Quantitative}

The EBPAS survey contains 36 questions rated on a 5 -point scale ranging from 0 ('not at all') to 4 ('to a very great extent'). Data analysis, including estimation of internal consistency (Cronbach's $\alpha$ ), was conducted according to criteria by Rye et al. ${ }^{16}$ EBPAS subscale scores were computed as the mean of the corresponding item ratings, as well as a total overall score representing respondent's broader attitudes towards evidence-based practice. Higher scores indicate a more positive attitude towards programme adoption.

\section{Qualitative}

Audio recordings of SSIs and FGDs were transcribed in Spanish and translated into English by study team members. One interview was excluded due to a technological error, leaving 81 SSIs for analysis. Deductive thematic analysis was conducted using NVivo V.12 for data organisation. Thematic data saturation was achieved at a sample size of 45 interviews; however, all interviews were analysed due to unit heterogeneity and to increase retrieval of salient items. ${ }^{27-29}$ We used a priori coding schemes using CFIR constructs adapted to patient safety. Three main coding headings were used (implementation barriers, implementation facilitators and contextual modifications) along with CFIR subcodes. Twenty-five per cent of the interviews were analysed by an additional study team member to ensure intercoder reliability, ensuring a minimum kappa statistic of 0.80 . Two team members coded the remainder of the transcripts.

Coded text was summarised by case memos, which were organised by objective and CFIR domain with illustrative quotes. Salience levels high/medium/ low $(\mathrm{H} / \mathrm{M} / \mathrm{L})$ were reported according to coding frequency percentile for all interviews $(\mathrm{H}=$ top 25 th and $\mathrm{L}=$ bottom 25 th). Dominant CFIR constructs were 
defined as constructs with high reporting frequency (top 25th percentile) in SSIs and FGDs. Valence levels were assigned to characterise the degree to which each CFIR construct impacted programme implementation according to the criteria of Damschroder and Lowery. ${ }^{30}$ Memos were reviewed for modification, adjudication and deliberation. A Guatemalan researcher reviewed all memos and illustrative quotes to ensure appropriate cultural and contextual interpretation. Data from FGDs were analysed using similar methods.

\section{Data integration}

Three different strategies were used to integrate qualitative and quantitative data: triangulation, complementarity and expansion. ${ }^{20}{ }^{31}$ First, we used the triangulation protocol described by Farmer et al to evaluate the convergence of qualitative and quantitative data (eg, do qualitative data agree with quantitative data regarding attitudes towards implementation?). ${ }^{32}$ A matrix was constructed to allow comparison of constructs from qualitative and quantitative data sets. Second, we compared data sets for complementarity to seek clarity on related findings using qualitative data to provide depth of understanding and quantitative data to provide breadth (eg, do qualitative data clarify and elaborate on the prevalence of attitudes observed in the quantitative results?). Third, we applied expansion to extend the range of understanding and to evaluate how qualitative analyses explained unanticipated quantitative findings (eg, do qualitative data expand on attitudes expressed or explain results observed in quantitative results?). Weight was attributed primarily to qualitative findings in all integration strategies.

To summarise dominant findings, all integrated data were categorised into broad themes with related constructs. All findings were evaluated for overall influence on implementation as either a barrier or facilitator, as well as for magnitude and strength.

\section{RESULTS}

\section{Participant characteristics}

Eighty-two staff from 11 units participated in the SSIs and EBPAS survey (table 1). The average number of key informants per unit was 7 (range 3-16). The majority of participants were physicians $(57 \%)$ with representation from nurses (27\%), clinical support $(10 \%)$ and administrative staff (6\%). More than half of respondents reported working in their specialty for more than 10 years, and most respondents were female (73\%). Many SSI participants (62\%) reported having little knowledge of patient safety concepts and practices.

Participants in the perioperative unit FGD included four physicians and 10 nurses. The neonatology FGD participants included three physicians, seven nurses and three support staff. Participants in both FGDs were predominantly female (perioperative $86 \%$, neonatology 92\%).
Table 1 Key informant characteristics

\begin{tabular}{lc}
\hline Key informant characteristics & $\mathrm{n}(\%)$ \\
\hline Total key informants & 82 \\
\hline Unit & \\
\hline Neonatology & $11(13.4)$ \\
\hline Outpatient & $5(6.1)$ \\
\hline Perioperative services & $16(19.5)$ \\
\hline HIV & $5(6.1)$ \\
\hline Infectious disease & $5(6.1)$ \\
\hline Emergency department & $12(14.6)$ \\
\hline Burn & $4(4.9)$ \\
\hline Infant inpatient & $3(3.7)$ \\
\hline Intermediate care & $5(6.1)$ \\
\hline Paediatric intensive care unit & $4(4.9)$ \\
\hline Paediatric inpatient & $3(3.7)$ \\
\hline Other & $9(11.0)$ \\
\hline Staff position & \\
\hline Physician (medical director, attending, resident) & $47(57.3)$ \\
\hline Nurse (nurse, nursing assistant, chief nurse) & $22(26.8)$ \\
\hline Clinical support (nutritionist, social worker, & $8(9.8)$ \\
psychologist, technician) & \\
\hline Administrative support (secretary, receptionist, & $5(6.1)$ \\
\hline logistics coordinator) & \\
\hline Years in specialty & $8(9.8)$ \\
\hline $1-2$ & $11(13.4)$ \\
\hline $3-4$ & $20(24.4)$ \\
\hline $5-10$ & $16(19.5)$ \\
\hline $11-20$ & $27(32.9)$ \\
\hline $21+$ & $22(26.8)$ \\
\hline Sex & $60(73.2)$ \\
\hline Male & \\
\hline Female & \\
\hline & \\
\hline
\end{tabular}

Key informants participated in both the semistructured interviews (SSIs) and completed the Evidence-Based Practice Attitude Scale-36 (EBPAS-36) survey. Staff who floated between units were captured under 'Other'.

\section{Quantitative data}

Scores on all EBPAS subscales indicated positive attitudes towards adoption of a safety programme and openness towards change in safety practices (figure 2). Staff reported favourable attitudes towards new interventions and use of safety manuals. Respondents reported that the fit of the programme, compatibility with their patients and feedback on performance were highly important. Staff expressed favourable attitudes towards organisational support and training on the patient safety programme. Concern for time and administrative burden associated with the programme was not high among respondents. EBPAS-36 had acceptable to excellent levels of internal consistency, with a mean Cronbach's $\alpha$ of 0.72 and subscale Cronbach's $\alpha$ between 0.54 and 0.85 (online supplemental file 5). 


\begin{tabular}{|c|c|}
\hline Scale & Abbreviated Sub-scale \\
\hline Openness & $\begin{array}{l}\text { Intervention developed by researchers } \\
\text { Like new intervention types } \\
\text { Will follow a treatment manual }\end{array}$ \\
\hline Fit & $\begin{array}{l}\text { Fit with your clinical approach } \\
\text { Had a say in how to use the EBP } \\
\text { Right for your patients }\end{array}$ \\
\hline Feedback & $\begin{array}{l}\text { Enjoy feedback on performance } \\
\text { Feedback helps me to be better } \\
\text { Supervision helps me to be better }\end{array}$ \\
\hline Appeal & $\begin{array}{l}\text { Colleagues happy with intervention } \\
\text { Get enough training to use } \\
\text { Makes sense }\end{array}$ \\
\hline Requirements & $\begin{array}{l}\text { Agency required } \\
\text { State required } \\
\text { Supervisor required }\end{array}$ \\
\hline $\begin{array}{l}\text { Organization } \\
\text { Support }\end{array}$ & $\begin{array}{l}\text { Continuing education credits provided } \\
\text { Ongoing support provided } \\
\text { Training provided }\end{array}$ \\
\hline Job Security & $\begin{array}{l}\text { Help me get a new job } \\
\text { Help me keep my job } \\
\text { Make it easier to find work }\end{array}$ \\
\hline Balance & $\begin{array}{l}\text { Overall competence is more important } \\
\text { Positive outcome is an art } \\
\text { Therapy is an art and a science }\end{array}$ \\
\hline Limitations & $\begin{array}{l}\text { Not individualized treatment } \\
\text { Patients with multiple problems } \\
\text { Too narrowly focused }\end{array}$ \\
\hline Divergence & $\begin{array}{l}\text { Clinical experience more important } \\
\text { Research based treatments not useful } \\
\text { Will not use manualized therapy }\end{array}$ \\
\hline Burden & $\begin{array}{l}\text { Can't meet other obligations } \\
\text { Don't have time to learn anything new } \\
\text { How to fit evidence-based practice }\end{array}$ \\
\hline Monitoring & $\begin{array}{l}\text { Looking over my shoulder } \\
\text { My work does not need to be monitored } \\
\text { Work without oversight }\end{array}$ \\
\hline
\end{tabular}

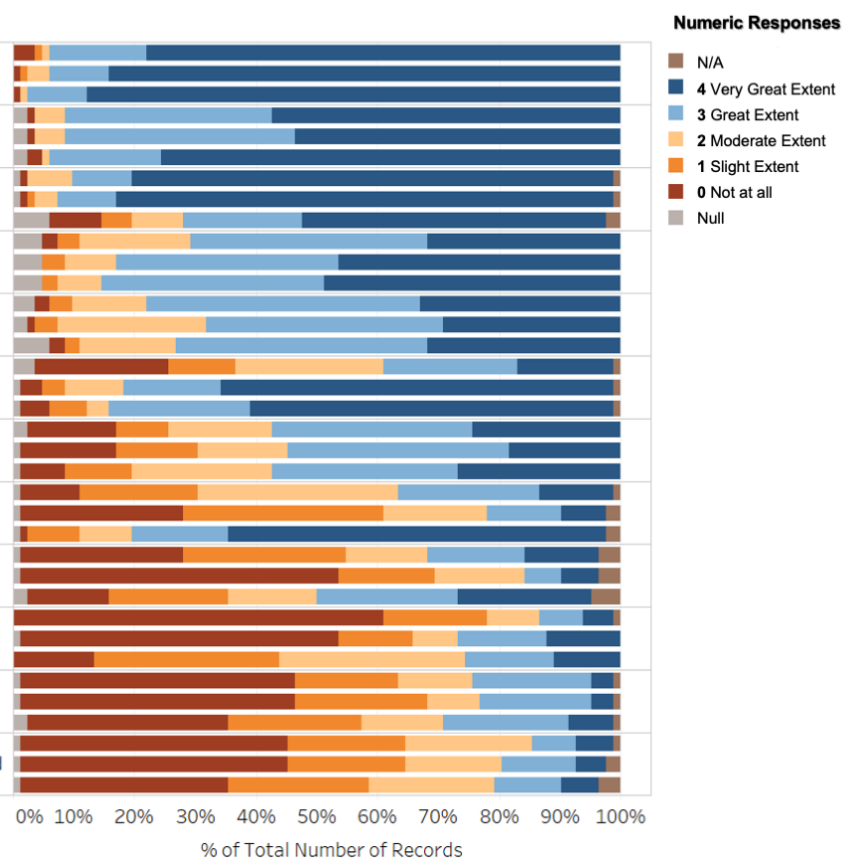

Figure 2 Evidence-Based Practice Attitude Scale-36 (EBPAS-36) Likert scale responses. Item responses range from 0 (strongly disagree) to 4 (strongly agree). Sample size ranges from 71 to 82 due to missing data.

\section{Qualitative data}

Semistructured interviews

We identified several CFIR domains and associated constructs that were dominant facilitators to programme implementation, including prioritisation of patient needs, tension for change and personal attributes. Dominant barrier constructs included limited organisational incentives, knowledge and beliefs, available resources, culture, external policies and incentives, and relative priority (figure 3).

Focus group discussions

Participants in the FGDs suggested several modifications to enhance programme implementation as discussed below. Participants confirmed several of the

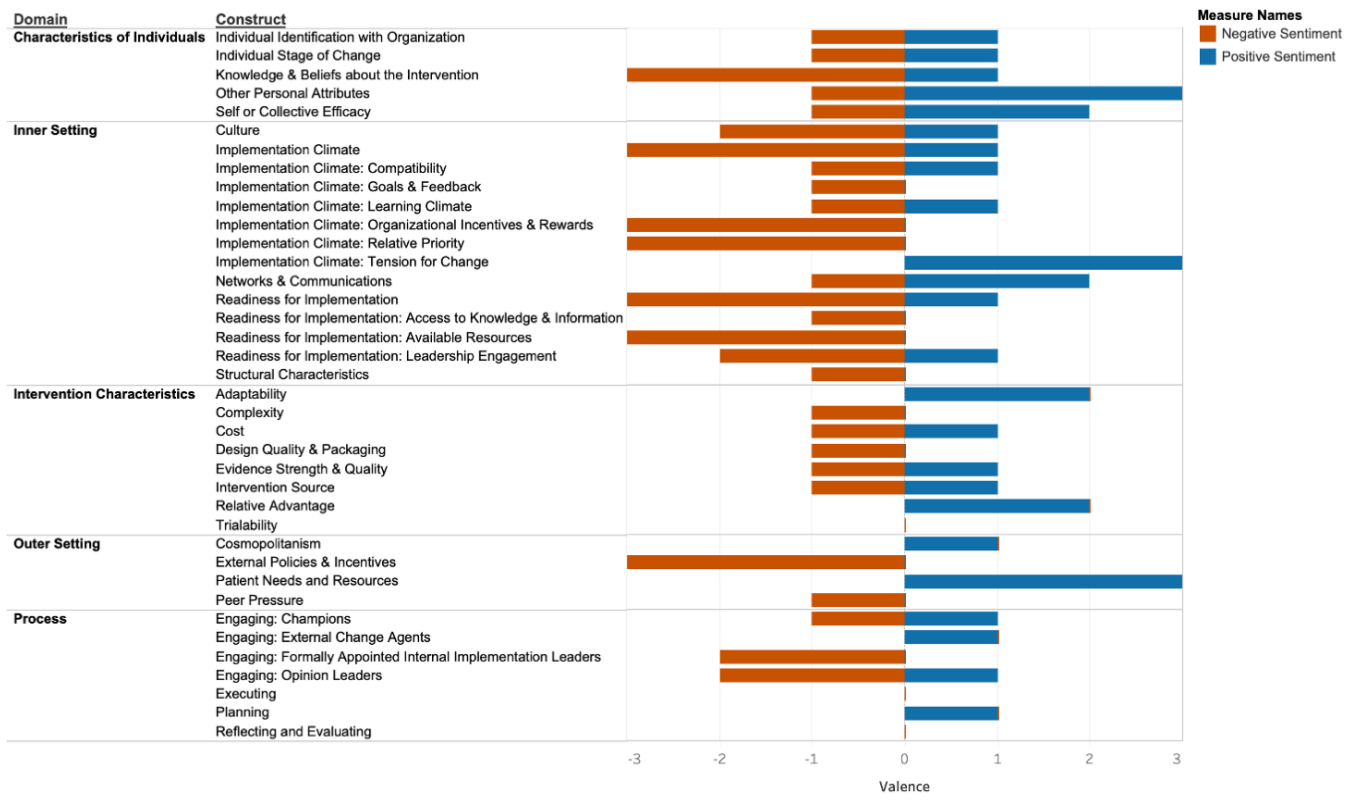

Figure 3 Valence of Consolidated Framework for Implementation Research (CFIR) constructs as represented in semistructured interviews (SSIs). Valence magnitude and direction are further delineated by influence to the implementation process as either a positive or negative sentiment. Valence criteria by Damschroder were adapted and applied as described: -3 , the construct is a strong negative influence on the implementation effort; -2 , the construct is a moderate negative influence on the implementation effort; -1 , the construct is a minor negative influence on the implementation effort; 0 , the construct is a neutral or no influence on the implementation effort. Alternatively, +1 , the construct is a minor positive influence on the implementation effort; +2 , the construct is a moderate positive influence on the implementation effort; +3 , the construct is a strong positive influence on the implementation effort. 
barriers and facilitators to implementation as identified in SSIs, including limited organisational incentives, low relative priority and a culture of blame. In terms of implementation execution, staff reported fragmented staff involvement, lack of accountability and competing unit priorities.

\section{Data integration}

We identified several facilitators and barriers to safety programme implementation from integration of quantitative and qualitative data as summarised by key themes (table 2). Supporting EBPAS subscales are indicated and supporting CFIR constructs are marked in italics with their associated salience levels high/ medium/low (H/M/L) reported according to coding frequency percentile.

Facilitator 1: patient-centredness

We found strong agreement in all data sets regarding favourable staff attitudes towards the ability for a safety programme to meet patient's needs. Patient needs (H) was identified as a primary construct to facilitate successful implementation. Commitment to a safety programme was tied to a desire to improve patient care rather than organisational identity. Staff who worked in units treating high-risk patients reported a strong fit between the safety programme and the need to avoid harm to patients. Some respondents discussed how a patient's vulnerabilities in education, income and health literacy could place them at greater risk for medical errors. Champions in the perioperative unit leveraged this by integrating patient needs into patient safety education and QI interventions. Consistent with qualitative findings, the EBPAS results reflected favourable attitudes towards programme fit for their patients (Subscale: Fit).

\section{Facilitator 2: staff receptivity and motivation}

A high degree of staff receptivity and motivation emerged through demonstration of complementarity. Within the domain Characteristics of Individuals, the construct other personal attributes $(\mathrm{H})$ emerged as a facilitator for implementation. Most respondents expressed strong motivation towards programme implementation, attributing their drive to caring for children. Similar sentiments were also represented in the Inner Setting domain as a strong tension for change $(\mathrm{H})$, including frustration with lack of awareness and accountability for medical errors. EBPAS scores complemented qualitative findings with high scores of staff openness towards evidence-based and new interventions (Subscale: Openness). Units engaged motivated front-line staff in unit-level working groups who modified safety interventions.

\section{Facilitator 3: desire for patient safety protocols}

Expansion of findings illustrated a desire for safety protocols as a driver for programme implementation.
Regarding the Intervention Characteristics domain, most participants perceived the safety programme as a relative advantage $(\mathrm{M})$ compared with prior absence of a programme. Staff valued use of a structured patient safety framework, including written guidelines and materials. Participants perceived the programme as having a high degree of adaptability (M) to unit resources and needs. EBPAS scores expressed favourable attitudes to openness to treatment manuals (Openness subscale item) and unfavourable views towards monitoring of their work (Subscale: Monitoring). Programme modifications in the perioperative unit included developing structured perioperative and intraoperative protocols and designated nurse champions $(\mathrm{H})$ to lead timeouts to enhance surgical checklist adherence during times when attending surgeons are not present.

Barrier 1: competing priorities amidst high patient care demands Competing priorities emerged by expansion as a dominant implementation barrier. Although EBPAS findings were only moderately suggestive of programme implementation being a burden (Subscale: Burden), SSI and FGD participants emphasised limited available time for programme implementation. Staff reported concerns about the lack of available resources $(\mathrm{H})$ needed to undertake programme implementation, specifically dedicated time and insufficient staff. Participants citing these challenges stressed the importance of integrating safety programmes into existing workflows. Participants reported the relative priority $(\mathrm{H})$ of patient safety programme was low due to competing priorities, and attributed this to a limited absorptive capacity at organisational and individual levels.

Barrier 2: lack of patient safety knowledge

A lack of knowledge about patient safety was identified as a critical implementation barrier. The majority of SSI participants had little knowledge of basic patient safety concepts, highlighting the barrier of knowledge and beliefs about the intervention $(\mathrm{H})$. Attending physicians reported understanding of safety tools used in local private hospitals but acknowledged inconsistent use of similar practices in public hospitals. Within the perioperative unit, the purpose of a patient safety checklist was understood by less than a quarter of study participants. In response, perioperative unit patient safety champions conducted staff training to ensure staff understood underlying key principles of checklist use and practices. In the neonatology FGDs, staff suggested that lack of knowledge of medical errors precluded error recognition and reporting. Subsequent modification of their error reporting system incorporated education and medical error examples to facilitate disclosure. 
Table 2 Key barriers and facilitators of patient safety programme implementation

\begin{tabular}{|c|c|c|c|c|}
\hline Key finding & $\begin{array}{l}\text { Integration } \\
\text { strategy }\end{array}$ & $\begin{array}{l}\text { CFIR domain: construct(s) } \\
\text { (qualitative data) }\end{array}$ & $\begin{array}{l}\text { EBPAS subscale: } \\
\text { item(s) (quantitative } \\
\text { data) }\end{array}$ & $\begin{array}{l}\text { Implementation } \\
\text { modifications }\end{array}$ \\
\hline \multicolumn{5}{|l|}{ Implementation facilitators } \\
\hline $\begin{array}{l}\text { Patient-centredness } \\
\text { '[PS] is very important because it } \\
\text { helps the patient in their recovery... } \\
\text { there are changes we have to do } \\
\text { because we know that it is going } \\
\text { to be for the good of the patient.' } \\
\text { (SSI 17) }\end{array}$ & $\begin{array}{l}\text { Triangulation } \\
\text { (congruence) }\end{array}$ & $\begin{array}{l}\text { Outer setting domain: patient needs } \\
\text { and resources (H) } \\
\text { Patients' needs known and } \\
\text { prioritised by staff. } \\
\text { Strong commitment to caring for } \\
\text { children. }\end{array}$ & $\begin{array}{l}\text { Fit subscale: right for } \\
\text { patients (3.55) }\end{array}$ & $\begin{array}{l}\text { Adaptation of programme to } \\
\text { fit patient needs through unit- } \\
\text { level working groups. } \\
\text { Integrate patient narratives } \\
\text { into educational efforts. }\end{array}$ \\
\hline $\begin{array}{l}\text { Staff receptivity/motivation } \\
\text { 'I think that all of the health } \\
\text { institutions should have a patient } \\
\text { safety program. That is quality care. } \\
\text { Obviously, in this country we are } \\
\text { very behind, that we don't have } \\
\text { a program like that. I think it's } \\
\text { important to do it for the safety of } \\
\text { the patient...' (SSI 70) }\end{array}$ & Complementarity & $\begin{array}{l}\text { Characteristics of individuals domain: } \\
\text { other personnel attributes }(\mathrm{H}) \\
\text { High degree of motivation to } \\
\text { improve patient care. } \\
\text { Inner setting domain: implementation } \\
\text { climate: tension for change }(\mathrm{H}) \\
\text { Physicians expressed the strongest } \\
\text { tension for change. }\end{array}$ & $\begin{array}{l}\text { Openness subscale: like } \\
\text { new intervention types } \\
\text { (3.74); interventions } \\
\text { developed by researchers } \\
\text { (3.63) }\end{array}$ & $\begin{array}{l}\text { Engage front-line staff in } \\
\text { programme implementation. } \\
\text { Provide meaningful feedback } \\
\text { to staff. }\end{array}$ \\
\hline $\begin{array}{l}\text { Desire for protocols } \\
\text { 'We don't have established }\end{array}$ & Expansion & $\begin{array}{l}\text { Innovation characteristics domain: } \\
\text { relative advantage (M) } \\
\text { - Programme advantageous }\end{array}$ & $\begin{array}{l}\text { Openness subscale: } \\
\text { will follow a treatment } \\
\text { manual (3.83) }\end{array}$ & $\begin{array}{l}\text { Provide treatment protocols to } \\
\text { support staff during times of } \\
\text { limited physician presence. }\end{array}$ \\
\hline
\end{tabular}

protocols for patient safety so there isn't a concrete way to ensure the safety of the patient. Each person does for the patient what they believe is better.' (SSI 16)

'You all [nurses] are the only staff here 24/7 and you should feel empowered to lead this [perioperative checklist].' (FGD 1) compared with prior absence of safety and quality efforts. Inner setting domain: readiness for implementation: access to knowledge and information (L)

- Desire for improved access to patient safety materials.

\section{Implementation barriers}

Competing priorities amidst high levels of patient care

....everyone has the motivation to improve things but not the time it takes to invest. They don't have [time] because they have to be doing different tasks at the same time and the main goal is to treat the patient, but I believe if the ultimate goal of better safety is to improve the care that [you] will give the patient, [then] it is necessary to devote a little time to this.' (SSI 23)

'There are so many other initiatives that it is hard for everyone to participate [in error reporting].' (FGD 15)

\section{Lack of knowledge about patient} safety concepts

'I think most do not believe in in this way of working because they do not know the results that can be obtained...education would be worth a lot in terms of patient safety.' (SSI 23)
Inner setting domain: readiness for implementation: available resources (H)

- Limited time for programme implementation.

- Low staff to patient ratios. Inner setting domain: implementation climate: relative priority $(\mathrm{H})$

- Competing priorities.
Monitoring subscale: my work does not need to be monitored (2.90); doesn't need someone looking over my shoulder (2.91)
Appeal subscale: makes sense (3.38); get enough training to use (3.32)
Burden subscale: don't have time to learn anything new (2.90); can't meet other obligations (2.84)

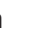

. 
Table 2 Continued

\begin{tabular}{|c|c|c|c|c|}
\hline Key finding & $\begin{array}{l}\text { Integration } \\
\text { strategy }\end{array}$ & $\begin{array}{l}\text { CFIR domain: construct(s) } \\
\text { (qualitative data) }\end{array}$ & $\begin{array}{l}\text { EBPAS subscale: } \\
\text { item(s) (quantitative } \\
\text { data) }\end{array}$ & $\begin{array}{l}\text { Implementation } \\
\text { modifications }\end{array}$ \\
\hline $\begin{array}{l}\text { Limited governance and oversight } \\
\text { 'We need political support and the } \\
\text { transmission of information. This } \\
\text { may be born of a committee or a } \\
\text { group that promotes this type of } \\
\text { measures to be generalized and } \\
\text { standardized not just at the hospital } \\
\text { level but also at the national level.' } \\
\text { (SSI 19) }\end{array}$ & Complementarity & $\begin{array}{l}\text { Process domain: formally appointed } \\
\text { implementation leaders (M) } \\
\text { Inner setting domain: readiness } \\
\text { for implementation: leadership } \\
\text { engagement (M) } \\
\text { Outer setting domain: external policies } \\
\text { and incentives (H) } \\
- \text { Limited governance at local, } \\
\quad \text { organisational and national levels. }\end{array}$ & $\begin{array}{l}\text { Requirements subscale: } \\
\text { supervisor required (3.04); } \\
\text { agency required (3.06); } \\
\text { state required (2.94) }\end{array}$ & $\begin{array}{l}\text { Report meaningful data at } \\
\text { organisational and national } \\
\text { levels to guide implementation } \\
\text { and policies. } \\
\text { Appoint patient safety and } \\
\text { quality committees. }\end{array}$ \\
\hline $\begin{array}{l}\text { Lack of organisational support } \\
\text { '... they always consider } \\
\text { [implementation] an extra load } \\
\text { that doesn't go in accordance to } \\
\text { a salary increase nor an additional } \\
\text { compensation.' (SSI 55) }\end{array}$ & Complementarity & $\begin{array}{l}\text { Inner setting domain: implementation } \\
\text { climate: organisational incentives and } \\
\text { rewards }(\mathrm{H}) \\
\text { Desire for support and improved } \\
\text { training to implement the } \\
\text { programme. }\end{array}$ & $\begin{array}{l}\text { Organisation support } \\
\text { subscale: continuing } \\
\text { education credits provided } \\
\text { (1.40); training provided } \\
\text { (3.30); ongoing support } \\
\text { provided (3.38) }\end{array}$ & $\begin{array}{l}\text { Provide compensation and/or } \\
\text { dedicated time. } \\
\text { Incentivise through goal- } \\
\text { sharing awards. }\end{array}$ \\
\hline $\begin{array}{l}\text { Poor culture and impact of human } \\
\text { factors } \\
\text { 'I think to start we should pursue } \\
\text { culture changes... and step by step }\end{array}$ & Expansion & $\begin{array}{l}\text { Inner setting domain: culture }(\mathrm{H}) \\
\text { Hierarchical culture. } \\
\text { 'Culture of blame'. } \\
\text { Process domain: engaging: opinion } \\
\text { leaders (M) }\end{array}$ & Not assessed & $\begin{array}{l}\text { Improve safety culture with } \\
\text { emphasis on teamwork and } \\
\text { 'just culture'. } \\
\text { Ensure transparency of safety } \\
\text { culture assessment data. }\end{array}$ \\
\hline
\end{tabular}

culture changes... and step by step

help the people understand why

safety culture is important.' (SSI 41)

'Staff would be more open to

documenting errors if they do not

have to report their name, they

would be less fearful.' (FGD 15)

Integration between qualitative and qualitative results was integrated using the following strategies: (1) triangulation—both sources reached the same conclusion (ie, congruence), (2) complementarity —interviews provided depth of understanding and surveys provided a breadth of information, (3) expansion — qualitative analyses explained unanticipated quantitative findings. Qualitative data from SSI and FGD represented by the CFIR. Dominant CFIR domains and constructs provided with associated salience level in parentheses. High/medium/low (H/M/L) saliency based on frequency percentile ( $H=$ top 25th and $L=$ bottom 25th). Related EBPAS subscales and items provided with mean scores in parentheses. Implementation modifications reflect recommendations in SSI and FGD data sets that were implemented in a real-time fashion or were prioritised for future modifications.

CFIR, Consolidated Framework for Implementation Research; EBPAS, Evidence-Based Practice Attitude Scale; FGD, focus group discussion; SSI, semistructured interview.

Barrier 3: limited governance and oversight

Complementarity revealed desires for increased leadership support. Staff emphasised the importance of external policies and incentives $(\mathrm{H})$ for programme implementation. Specifically, they reported the need for hospital and national patient safety policies, outcome data and financial support. Respondents shared that policies were essential to foster accountability for medical errors, which in turn would facilitate implementation. Staff considered national governance less important compared with unit and hospital-level governance (Subscale: Requirements).

Barrier 4: lack of organisational support

EBPAS results showed favourable attitudes towards adoption of practices that increased organisational support, and SSI and FGD participants confirmed that implementation could not be sustainable without increased incentives. Despite strong desires for change, staff cited that organisational incentives $(\mathrm{H})$ such as dedicated time, training and rewards were not allocated adequately. To address the lack of organisational incentives, staff suggested incorporating non-financial compensation for safety activities, such as staff recognition and honorary rewards.

Barrier 5: culture and human factors

Although not assessed in EBPAS, qualitative data suggested a strong negative influence of cultural and hierarchical dynamics. Many respondents associated implementation difficulty with poor organisational culture and disconnected leadership. Some staff highlighted the importance of a strong safety culture, and also discussed the strong influence of unit chiefs (Engaging: Opinion Leaders (M)) in establishing the programme as a priority. Staff in the neonatology unit attributed the low rates of medical error reporting to a culture of blame $(\mathrm{H})$ and lack of knowledge $(\mathrm{H})$ affecting error identification. In response to these 
barriers, the neonatology unit modified their error reporting mechanism to include a private drop box, as well as a WhatsApp communication thread to relay concerns and results to staff.

\section{DISCUSSION}

We identified several challenges to implementation of a comprehensive patient safety programme in Guatemala. Use of the CFIR framework with a mixed methods study design allowed us to identify key barriers and facilitators to implementation that are contextualised to this setting. Embedding an implementation analysis early during programme deployment allowed for modification to enhance successful implementation. Our work demonstrates the value of understanding how multiple stakeholders interact within a complex health system organisational structure to support safety programme implementation. These findings expand our understanding of the organisational, contextual and cultural factors which impact safety programme implementation in an LMIC, and should help efforts to improve patient safety in similar low-resource settings.

We found that use of a structured framework such as CFIR for research tool design and data analysis is essential to evaluate the complex challenges to safety programme implementation in Guatemala. Implementation challenges differ between LMICs and highincome settings due to differences in cultural or social norms, disease burden and financial resources, among other determinants. ${ }^{9}$ Use of structured frameworks can support systematic evaluation of these implementation challenges, although any framework requires adaptation or contextualisation to a given LMIC setting. For example, we found that organisation of our data using CFIR allowed us to identify key gaps within individual domains (eg, knowledge about patient safety within Characteristics of Individuals domain) as well as discern themes that presented across multiple domains of influence (eg, need for increased governance across Inner Setting, Outer Setting and Process domains). Means $e t$ al have proposed that an additional domain called 'Characteristics of Systems' be added to the CFIR to increase its compatibility for use in LMICs to account for the relationship between key system characteristics. ${ }^{9}$ We would support this CFIR modification, as interactions between national health system leaders and hospitals in Guatemala suggest that both the outer and inner settings influence how each other system evolves over time.

Although implementation efforts around the world focus on closing the 'know-do' gap, our findings suggest there is also a 'knowledge gap' in understanding patient safety in Guatemala. For example, most respondents were unable to articulate the role of patient safety tools and QI interventions or identify specific benefits for patients. Knowledge gaps regarding patient safety have been reported in other LMICs among staff and students. ${ }^{33-35}$ Our findings reveal that limited familiarity with patient safety concepts impacts implementation but also may contribute to lack of understanding of the value of a patient safety programme itself (relative priority construct) and programme adherence. These data suggest that safety efforts must go beyond use of discrete safety tools alone, and require educational systems to improve staff knowledge about basic patient safety principles. Educational efforts should emphasise the application of 'systems thinking', integrating the concepts of fallibility and 'just culture' in clinical operations. ${ }^{3637}$

Staff attitudes reflected a high degree of receptivity to patient safety efforts, pointing to psychological readiness to implement safety programmes in their setting. However, despite this strong desire to improve patient safety, we found a lack of organisational readiness, threatening successful implementation. These findings are consistent with existing literature illustrating that soft factors (eg, staff motivation, leadership, culture) alone are insufficient to drive sustained implementation of new programmes. ${ }^{4839}$ Over time, strategies that focus only on leveraging staff motivation without appropriate attention to improving workload and organisational incentives risk not being sustained. As argued by Weiner, receptivity is a necessary but not sufficient condition for implementation readiness, suggesting that efforts to link staff receptivity with concrete organisational support are required for implementation. $^{40}$

We found that there is a need for incentives to sustain safety practices such as expansion of hospital and national patient safety policies, access to highquality outcome data and financial support. Some of these challenges have been highlighted around the world recently as health systems struggle during the COVID-19 pandemic, which has revealed existing gaps in patient safety systems, low capacity for organisational change and poor teamwork. ${ }^{104142}$ As well, we suggest that the barriers to safety programme implementation in Guatemala are similar to those seen in high-income settings at the early stages of building safety programmes, such as the need to first develop a strong safety culture and leadership engagement. This is not to say that processes seen in high-income countries are either necessary or even optimal in an LMIC, rather that complexity of approaches to patient safety is a natural outgrowth of safety practices around the world.

Given these contextual challenges, we highlighted several implementation priorities of a comprehensive patient safety strategy that are essential to facilitate successful uptake of a safety programme (online supplemental file 6). Critical initial components of this approach should focus on improving patient safety knowledge, addressing human factor barriers through building a strong safety culture and prioritisation by leadership. Intermediate objectives should include 
capacity building, leadership training and integration of programme components into existing workflows. Longer term efforts should prioritise embedding educational curricula and monitoring of safety metrics. Particularly for the complex care of children, all these elements are essential for sustained safety programme implementation given the complex physiological, equipment and training needs for high-quality paediatric care.

There are several strengths of our study. First, a mixed methods approach enables integration of quantitative and qualitative data to evaluate the complex implementation challenges in low-resource settings. ${ }^{7}$ Use of a structured framework such as the CFIR can identify the multidimensional attributes of implementation challenges, while the dual use of CFIR and EBPAS captures a broad range of characteristics. $^{9}{ }^{43}$ Second, our report emphasises the importance of perspectives from front-line staff, which provides insights on day-to-day implementation challenges of health programmes. Third, our study reinforces the value of embedding an implementation analysis early in programme deployment, such that design or implementation process can be modified in a realtime fashion to evaluate implementation success and support a culture of continuous learning and change. ${ }^{44}$

Despite these strengths, our study is limited by several challenges. First, this research was conducted at a single institution, limiting the transferability of findings. Second, our analysis did not include patient or family perspectives. Third, our findings did not focus on clinical outcomes, as this requires extended collection of clinical data and is beyond the capacity of our current analysis. Fourth, although the EBPAS performed well in terms of internal consistency, further tool validation in low-resource settings is required. Fifth, our interview guides were informed by select CFIR constructs based on our prior studies, raising the possibility for selection bias. Sixth, limited familiarity with patient safety concepts may have impacted respondents' perceptions leading to low salience of certain constructs, such as those related to intervention characteristics. Future research should include outcome evaluations to test the relationship between construct salience and impact on outcome metrics.

In conclusion, our evaluation identified several dominant themes which affect the implementation of a patient safety programme in Guatemala. Leadership at all levels should work together to strategically address implementation barriers, such as gaps in patient safety education, limited governance, poor safety culture and lack of incentives. Equally, facilitators to programme implementation, such as a strong commitment to caring for children and high staff receptivity, should be leveraged for programme design and implementation. The use of comprehensive patient safety programmes is critical as QI efforts around the world move beyond individual patient safety tools towards system-wide safety practices. Embedding an implementation analysis early during safety programme deployment itself can facilitate programme modification to enhance successful uptake.

Twitter J Bryan Sexton@JBryanSexton1

Contributors Study design: HR, RLM, JNB, DB, JBS, EC. Data collection: BJH, MP, AA, IS, MOB, SM, CCR, AD, AT, HR. Data analysis: BJH, MP, AA, IS, MOB, SM, CCR, AD, AT, HR. Data interpretation: BJH, AA, IS, HR, JNB. Manuscript writing: BJH, HR. Manuscript revision: HR, BJH, AA, MP, IS, JNB, EC, RLM.

Funding This research was supported by the Fogarty International Center, National Institutes of Health, USA, under award number R03TW010670.

Competing interests None declared.

Patient consent for publication Not required.

Provenance and peer review Not commissioned; externally peer reviewed.

Data availability statement Data are available upon reasonable request. To protect the confidentiality of study participants, data are available upon reasonable request to the corresponding author.

Supplemental material This content has been supplied by the author(s). It has not been vetted by BMJ Publishing Group Limited (BMJ) and may not have been peer-reviewed. Any opinions or recommendations discussed are solely those of the author(s) and are not endorsed by BMJ. BMJ disclaims all liability and responsibility arising from any reliance placed on the content. Where the content includes any translated material, BMJ does not warrant the accuracy and reliability of the translations (including but not limited to local regulations, clinical guidelines, terminology, drug names and drug dosages), and is not responsible for any error and/or omissions arising from translation and adaptation or otherwise.

Open access This is an open access article distributed in accordance with the Creative Commons Attribution Non Commercial (CC BY-NC 4.0) license, which permits others to distribute, remix, adapt, build upon this work noncommercially, and license their derivative works on different terms, provided the original work is properly cited, appropriate credit is given, any changes made indicated, and the use is noncommercial. See: http://creativecommons.org/licenses/by-nc/4. $0 /$.

\section{ORCID iD}

Bria J Hall http://orcid.org/0000-0002-4354-1590

\section{REFERENCES}

1 Khan MA, Soteriades ES, King J, et al. Global trends and forecast of the burden of adverse effects of medical treatment: epidemiological analysis based on the global burden of disease study. Cureus 2020;12:e7250.

2 Sachs J, Schmidt-Traub G, Kroll C. SDG index and dashboards report 2018: global responsibilities. Glob Responsab Implement Goals 2018.

3 World Health Organization. Patient safety: making health care safer, 2017.

4 Kruk ME, Gage AD, Arsenault C, et al. High-quality health systems in the sustainable development goals era: time for a revolution. Lancet Glob Health 2018;6:e1196-252.

5 Schwartz SP, Rehder KJ. Quality improvement in pediatrics: past, present, and future. Pediatr Res 2017;81:156-61.

6 Durlak JA, DuPre EP. Implementation matters: a review of research on the influence of implementation on program outcomes and the factors affecting implementation. Am J Community Psychol 2008;41:327-50. 
7 Ridde V. Need for more and better implementation science in global health. BMJ Glob Health 2016;1:e000115.

8 Damschroder LJ, Aron DC, Keith RE, et al. Fostering implementation of health services research findings into practice: a consolidated framework for advancing implementation science. Implement Sci 2009;4:50.

9 Means AR, Kemp CG, Gwayi-Chore M-C, et al. Evaluating and optimizing the consolidated framework for implementation research (CFIR) for use in low- and middle-income countries: a systematic review. Implement Sci 2020;15:1-19.

10 Hirschhorn L, Smith JD, Frisch MF, et al. Integrating implementation science into covid-19 response and recovery. BMJ 2020;369:m1888.

11 Rice HE, Lou-Meda R, Saxton AT, et al. Building a safety culture in global health: lessons from Guatemala. BMJ Glob Health 2018;3:e000630.

12 Lou-Meda R, Méndez S, Calgua E, et al. Developing a national patient safety plan in Guatemala. Rev Panam Salud Publica 2019;43:1.

13 Sico IP, Hall BJ, Aguilar-González A, et al. Implementation analysis of a perioperative patient safety program in Guatemala. World J Surg 2020;44:2131-8.

14 Palinkas LA, Horwitz SM, Chamberlain P, et al. Mixedmethods designs in mental health services research: a review. Psychiatr Serv 2011;62:255-63.

15 Kirk MA, Kelley C, Yankey N, et al. A systematic review of the use of the consolidated framework for implementation research. Implement Sci 2016;11:72.

16 Rye M, Torres EM, Friborg O, et al. The evidence-based practice attitude Scale-36 (EBPAS-36): a brief and pragmatic measure of attitudes to evidence-based practice validated in US and Norwegian samples. Implement Sci 2017;12:44.

17 De Paúl J, Indias S, Arruabarrena I. Adaptation of the evidence-based practices attitude scale in Spanish child welfare professionals. Psicothema 2015;27:341-6.

18 van Sonsbeek MAMS, Hutschemaekers GJM, Veerman JW, et al. Psychometric properties of the Dutch version of the evidence-based practice attitude scale (EBPAS). Health Res Policy Syst 2015;13:1-12.

19 Magidson JF, Lee JS, Johnson K, et al. Openness to adopting evidence-based practice in public substance use treatment in South Africa using task shifting: caseload size matters. Subst Abus 2018;39:162-6.

20 Palinkas LA, Aarons GA, Horwitz S, et al. Mixed method designs in implementation research. Adm Policy Ment Health 2011;38:44-53.

21 Johnston BE, Lou-Meda R, Mendez S, et al. Teaching patient safety in global health: lessons from the Duke global health patient safety fellowship. BMJ Glob Health 2019;4:e01220.

22 WHO. Process of translation and adaptation of instruments, 2010.

23 Sousa VD, Rojjanasrirat W, Translation RW. Translation, adaptation and validation of instruments or scales for use in cross-cultural health care research: a clear and user-friendly guideline. J Eval Clin Pract 2011;17:268-74.

24 Tong A, Sainsbury P, Craig J. Consolidated criteria for reporting qualitative research (COREQ): a 32-item checklist for interviews and focus groups. Int $J$ Qual Health Care 2007;19:349-57.
25 Aspden P, Corrigan JM, Wolcott J. Patient safety: Acheiving a new standard of care. National Academies Press (US), 2004.

26 Saluja S, Silverstein A, Mukhopadhyay S, et al. Using the Consolidated Framework for Implementation Research to implement and evaluate national surgical planning. BMJ Glob Health 2017;2:e000269.

27 Namey E, Guest G, McKenna K, et al. Evaluating bang for the buck. Am J Eval 2016;37:425-40.

28 Guest G, Namey E, McKenna K. How many focus groups are enough? building an evidence base for nonprobability sample sizes. Field methods 2017;29:3-22.

29 Weller SC, Vickers B, Bernard HR, et al. Openended interview questions and saturation. PLoS One 2018;13:e0198606.

30 Damschroder LJ, Lowery JC. Evaluation of a large-scale weight management program using the consolidated framework for implementation research (CFIR). Implement Sci 2013;8:51.

31 Cresswell J, Clark P V. Designing and conducting mixed method research. Thousand Oaks, CA: Sage Publications, 2007.

32 Farmer T, Robinson K, Elliott SJ, et al. Developing and implementing a triangulation protocol for qualitative health research. Qual Health Res 2006;16:377-94.

33 Ogunlusi JD, Yusuf MB, Ogunsuyi PS, et al. Awareness and Use of Surgical Checklist among Theatre Users at Ekiti State University Teaching Hospital, Ado-Ekiti, Nigeria. Niger J Surg 2017;23:134.

34 Nwosu ADG, Onyekwulu FA, Aniwada EC. Patient safety awareness among 309 surgeons in Enugu, Nigeria: a crosssectional survey. Patient Saf Surg 2019;13:33.

35 Nabilou B, Feizi A, Seyedin H. Patient safety in medical education: students' perceptions, knowledge and attitudes. PLoS One 2015;10:e0135610.

36 Iedema R. Attitudes toward error disclosure need to engage with systems thinking. Jt Comm J Qual Patient Saf 2010;36:99-100.

37 Tetuan T, Ohm R, Kinzie L, et al. Does systems thinking improve the perception of safety culture and patient safety? J Nurs Regul 2017;8:31-9.

38 Ruelas E. Health care quality improvement in Mexico: challenges, opportunities, and progress. Proc 2002;15:319-22.

39 Coles E, Anderson J, Maxwell M, et al. The influence of contextual factors on healthcare quality improvement initiatives: a realist review. Syst Rev 2020;9:94.

40 Weiner BJ. A theory of organizational readiness for change. Implement Sci 2009;4:67.

41 Tannenbaum SI, Traylor AM, Thomas EJ, et al. Managing teamwork in the face of pandemic: evidence-based tips. BMJ Qual Saf 2021;30:59-63.

42 Staines A, Amalberti R, Berwick DM, et al. COVID-19: patient safety and quality improvement skills to deploy during the surge. Int J Qual Health Care 2021;33.

43 Rojas Smith L, Ashok M, Morss Dy S. Discussion. In: Contextual Frameworks for Research on the Implementation of Complex System Interventions [Internet]. Agency for Healthcare Research and Quality (US), 2014.

44 Churruca K, Ludlow K, Taylor N, et al. The time has come: embedded implementation research for health care improvement. J Eval Clin Pract 2019;25:373-80. 